\title{
Toward a Platform of Human-Like Fingertip Model in Haptic Environment for Studying Sliding Tactile Mechanism
}

\author{
Van Anh Ho*
}

\author{
Shinichi Hirai**
}

\begin{abstract}
Human fingertip and its derivatives in robotics possess an inhomogeneous structure with skin, tissue, bone, and nail; which causes troublesome for construction of a precise model that can represent almost mechanical characteristics, especially during sliding motion. Commonly, models in a virtual haptic environment are frictionless, thus attempts to display tactile sensations are still remained unsolved. We have proposed a Beam Bundle Model for modeling of a human fingertip during pushing and sliding action with friction, especially stickto-slip transition, to overcome mentioned issues. In order to construct its three-dimensional non-homogeneous structure, we took sequential series of magnetic resonant images, which bring consecutive cross-sectional layers of the human fingertip with distribution of skin, tissue, bone, and nail. Simulation results show a twofold aspect. Firstly, it can generate not only normal force distribution caused by pushing, but also response of friction force during sliding. Secondly, and more interestingly, the model dynamically produces localized displacement phenomenon on the contact area during stick-to-slip phase, which indicates how slippage erodes the contact area before the total slippage of the fingertip occurs. This research helps to assess the sliding process of the human fingertip, including how and when the slippage occurs on the contact surface. This model may also act as a platform for studying tactile perception of the fingertip, as well as being a virtual environment for haptic interfaces.
\end{abstract}

\section{INTRODUCTION}

The development of smart electronic devices has enhanced interest in touching and sliding actions of human fingertips. Moreover, interactions between human fingertips and machines require a novel sensing system that detects diverse actions, such as pushing, squeezing, and especially sliding. In the virtual world of a haptic system, a successful tactile exploration requires the creation of a significant sensation of realness to the user through virtual objects [1].

Modeling bio-mimetically structured fingertips has been an interesting issue in robotics for years. These models started with a model of a homogeneous soft fingertip, with the fingertips being in simple geometrical shapes, such as a cylinder or hemisphere. Using nonlinear finite element analysis (FEA), the contact mechanics of a hemispherical fingertip were assessed, focusing primarily on normal contact that causes a pressure distribution profile over the contact zone to verify power-law theory in material mechanics [2]. Three dimensional FE models of human and monkey fingertips, with five layers of epidermis, dermis, and bone, have been developed to investigate the roles of the epidermis layer, as

\footnotetext{
*V.A. Ho is with the Advanced Technology R\&D Center, Mitsubishi Electric Corporation, Japan. Email: hoanhvan@gmai l . com

**S. Hirai is with the Department of Robotics, Ritsumeikan University, Japan. Email: hirai@se.ritsumei.ac.jp
}

well as the stiffness of the other layers, in representations of a line load on the fingertip [3]. Although the model perfectly fitted experimental data, calculation times were high. In addition, this model ignored friction, as well as dynamic responses during sliding motion. A more complicated model of a human fingertip with accurate geometries has been proposed [4]-[6] to study the deformation of a fingertip on a flat plate. These models, however, were concerned only with static models that predict stress-strain when making contact; neither could be used to evaluate dynamic changes in stressstrain over time. During sliding actions, a fingertip must feel surfaces and act correspondingly to prevent grasping or manipulation. Although a closed form has been built for modeling manipulation with sliding fingers, that model was limited to assessing quasi-static simulation and homogeneous fingertips, but could not assess details of slippage on the contact area [7]. A friction display system was recently introduced for a virtual environment, using a simplified model of a virtual spring during transition from slip to stick, and vice versa [1]. While this model can evaluate the characteristics of a human fingertip, such as switching between sticking and slipping and oscillations in slip phase, it could not determine the mechanism by which partial slips occur on the contact area during sticking phase, which is crucial in assessing slip perception. A combination of Signorini's contact law and Coulomb's friction law with algorithms for linearity has been proposed [8], as has haptic rendering of interacting deformable objects. Although these findings provide realtime haptic feedback, it is limited to homogeneous linear elastic objects.

Based on our previous work, [9] we propose using a Beam Bundle Model BBM) to model a human fingertip. The new contribution is detailed in Section III-A. Magnetic Resonant images (MRI) of a volunteer's index fingertip were used to construct a mathematical model of the fingertip's structure. We first characterized the parameters of pre-sliding on a real human finger to determine its inherent characteristics. We later constructed a simulated model, and we succeeded in representing localized displacements (also referred to as skin local stretch) during the pre-sliding phase of a fingertip. This assessment is considered crucial for evaluating stick/slip events on the contact pad. Our findings can be utilized not only in haptic sensation, but also in developing sensors to detect slippage.

\section{Human Fingertip Frictional Characteristic}

All current robotic fingertips differ markedly from human fingertips in structure and function, since the latter 


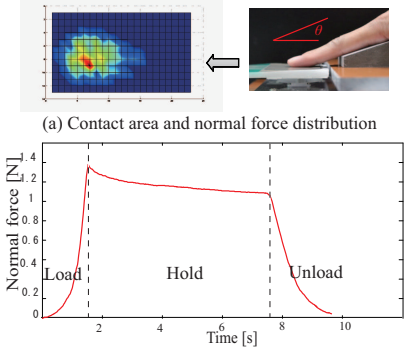

(b) Normal force response during load-unload test

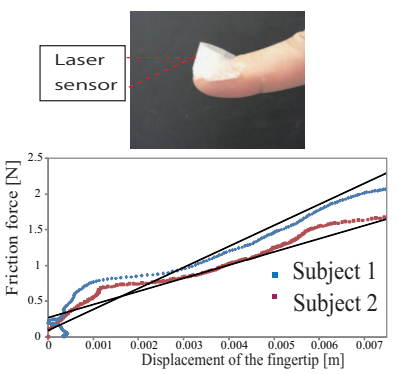

(c) Friction vs. finger displacement during stick phase

Fig. 1. Experimental setup and force-related results

are composed of complex and inhomogeneous structures. Thus, it is necessary to characterize the slipping action of a human fingertip, both to assess the kinds of phenomena that occur during contact/slide interactions and to introduce these realistic issues into our proposed model.

We designed an experiment to evaluate the mechanic characteristics of human fingertips. Subjects were asked to push and slide their index fingertips on a rigid, transparent surface. The translational movement of the finger was tracked by a laser range sensor with a resolution of $0.1 \mathrm{~mm}$, while the three components of force applied to the surface were recorded by a 3-degree of freedom (dof) loadcell. We used a pressure distribution sensor to assess the distribution of normal forces when pushing the finger. A high speed camera was used to determine detailed deformations of the contact area of the fingertip during sliding. As mentioned above in Section I, we focused on the pre-sliding activities of the human fingertip.

\section{A. Relaxation of Normal Force}

Fig. 1 (a) shows the normal force distribution on the contact area when a fingertip is pushed at a specific contact depth. In contrast to the force distribution of typical robotic fingertips, such as cylindrical and hemispheric fingertips, this distribution is asymmetrical, and unpredictable if based only on the boundary geometric shape. Moreover, the normal force responses during the loading, holding, and releasing phases vary significantly as the rate (of loading/unloading) changes (Fig. 1(b)). Moreover, relaxation occurs when a fingertip maintains its position at a specific contact depth. Thus, it is not sufficient to introduce elasticity alone to the model of a human fingertip [1]. Rather, a nonlinear viscoelastic model may predict a the relationship between force and displacement of the pulp of a fingertip [10]-[11].

\section{B. Spring-Like Behavior}

Some friction models [16] are based on a spring-like relationship between frictional force and the displacement of an object, especially soft objects, during the sticking phase. We have investigated this characteristic of human fingertips by asking subjects to start to slide their fingers slowly enough for fingertip movement to be captured by the laser displacement sensor, while the loadcell measures the friction force acting on it. A light-weight shield was attached
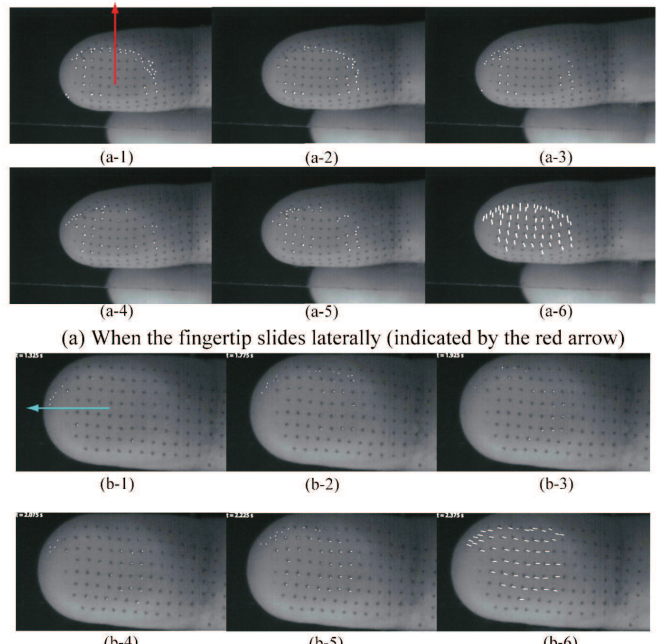

(b) When the fingertip slides forward (indicated by the blue arrow)

Fig. 2. Movements on the contact area during the stick phase. White dots/bars indicated the slipped points on the contact pad

to the nail of each fingertip to assure the correct reflection of the laser beam from the displacement sensor (see the inset picture in Fig. 1(c)). We observed a sticking phase prior to the gross sliding of the fingertip, during which the fingertip gives way while the contact area still sticks, causing an increase in friction [1].

\section{Localized Displacement during Stick Phase}

Considerable experimental research has assessed partial movements of the contact area during the pre-sliding or sticking phase of soft robotic fingertips ([7]-[9]). This phenomenon is also thought to occur on human fingertips, thanks to their soft dermis and curved shape. In this experiment, we attempted to verify this phenomenon by using a high speed camera to assess the movements of dots marked on the skin of the contact area of human fingertips. After marking black dots onto subjects' fingertips, the subjects were asked to slide their fingers gradually from zero along different directions, while attempting to maintain velocity and normal force. We employed an optical tracking method [12] to track the contact points. Fig. 2 shows the tracking of the dots on the contact area, with the movements of the dots, as indicated by white bars over time, during the pre-slide phase. We observed localized displacements of the contacting dots in the sticking phase. These displacements start from the boundary areas of the contact pad and then propagate to inner areas, with the slip phase occurring only when these partial movements are dominant all over the contact pad. Moreover, the propagation of partial movement differed according to the direction of the slide. To date, no analytical model was able to tackle this issue in its closed form, since the transition between the stick-slip states of each contact point had an inherent discontinuity. Our findings allowed a model in which generalized displacements of contact points could be derived with the introduction of stick-slip transition. 
Thus, to fully describe the inherent characteristics of a human fingertip in the model, the issues mentioned above must be included, at least in part.

\section{BeAm Bundle Model of Human FingertiP}

\section{A. Limit of the Previous Model}

Previously [9], we proposed a model to simulate deformations of hemispherical soft fingertips. In this model, the soft fingertip is necessarily elastic and homogeneous with a predetermined geometrical shape. Deformation of the fingertip under normal and tangential loads were modeled through virtual elastic compressible and bendable cantilevers. The contact area was meshed with a viscoelastic model according to finite element theory. This model allowed a simulation of sliding motion, during which the responses of normal and frictional forces could be observed. Moreover, simulation also produced stick/slip on the contact area, helping to assess how and when the slippage occurred on the contact surface. This model, however, is insufficient for modeling non-homogeneous, non-elastic fingertips with irregular geometrical shapes. Also, we had ignored the dynamic response of normal force during the load/unload phase.

\section{B. Construction of Human Fingertip Using MR Images}

A 3 Tesla MRI system was used to accurately measure the three-dimensional (3D) internal and external geometries of a human fingertip. Cross-sectional MR images of an index finger were obtained from a 25-year-old adult male volunteer with no history of finger disease. A total of 32 images, $120 \times 120 \mathrm{~mm}^{2}$ in size, with $1.2 \mathrm{~mm}$ slice thickness and $512 \times 512$ matrices (pixel size $0.23 \times 0.23 \times 1.2 \mathrm{~mm}^{3}$ ) were acquired using a fast gradient echo (fgre) sequence with $9.8 \mathrm{~ms}$ repetition time (TR), $4.2 \mathrm{~ms}$ echo time (TE) and 30 flip angle. These images of consecutive cross-sectional layers represented the volume of the fingertip. We were able to assess the distribution of layers, such as skin, tissue, bone, and nail position, for each image, as illustrated in Fig. 3 .

It is necessary to assess the exact distribution of constructed layers of the fingertip. We utilized image processing functions in OpenCV to extract boundaries of skin, bone, and nail. Since MR signals are mainly derived from protons of water molecules in body tissues, we were not able to extract the shape of the nail, which is dry; rather, we were able only to extract the boundary of the tissue on which the nail sits. Each boundary was formed by a group of points, which were afterward interpolated into a curve (see Fig. 4(a)). Thus, for each image, we were able to collect four fitted curves, one each for the skin, lower bone, upper bone, and nail (Fig. 4(a)). By repeating this process on all images, the three-dimensional geometrical shape of the fingertip could be constructed.

\section{Construction of BBM for Human Fingertip}

After obtaining boundary curves of the structure of a human fingertip, we had to fill in the remaining volume with virtual beams, which is similar to an elastic cantilever [9]. These beams have two ends constrained by an upper

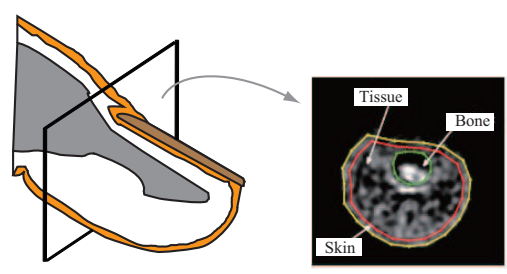

Fig. 3. MR images of consecutive cross-sectional layers of a human fingertip, showing the distribution of skin, phalanx, and nail.

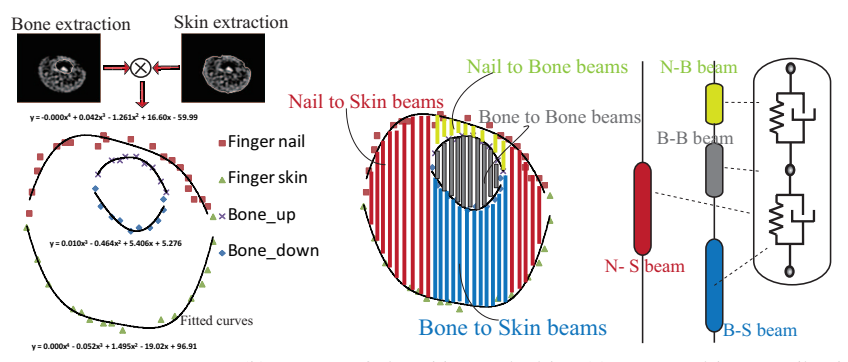

(a) Structure extraction (b) Groups of virtual beams inside (c) Beams with generalized

$$
\text { the fingertip Voigt element }
$$

Fig. 4. Structure of human fingertip from MRI.

(nail) and lower (skin) boundary. Each virtual beam is cylindrical in shape with geometrical properties, such as height, predetermined based on known fitted curves of nail, bone, and skin. Since layers of MR images have a pitch of $1.2 \mathrm{~mm}$ along the volume of the fingertip, and to fit beams into the volume of the fingertip, each beam had a crosssectional diameter of $1.2 \mathrm{~mm}$. Thus, the distance between basic axes of two neighboring beams was also $1.2 \mathrm{~mm}$.

To reflect the relaxation mentioned in Section II-A, we used a generalized Voigt model that includes two Voigt elements to assign each beam. Each element had a viscoelasticity characterized by a spring and a damper connected in parallel, similar to findings showing that fingertip pulp responds as a viscoelastic material [10]. After filling in with virtual beams (Fig. 4(b)), there were two main groups of beams, those attached to the nail and skin (N-S) and those attached to the nail through bone (N-B-S). Beams in the latter group consisted of three separated beams connected in series: N-B, B-B, and B-S (Fig. 4(c)). As a result, virtual beams were not necessarily homogeneous as in [9]. Using these types of non-homogeneous beams, we could fill in any soft fingertip, regardless of how complicated its structure.

If a fingertip was pushed vertically onto a rigid flat surface, causing a set of contact boundary points on the skin, the, using finite element analysis, the contact area (or skin) could be covered with a set of triangles (Fig. 5(a)), with the stressstrain relationship of each triangle represented by a Voight element that included a spring and a damper connected in parallel (Fig. 5(b)). Each triangle would have three nodes attached to the corresponding free ends of three beams belonging to either the N-S or B-S group (see Fig. 5(c)). The beams of each triangle could make two types of contribution: partly covering and superposition. The former occurs more 


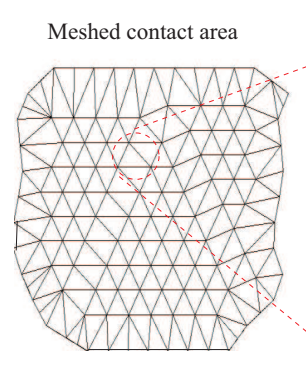

(a) Meshed contact area

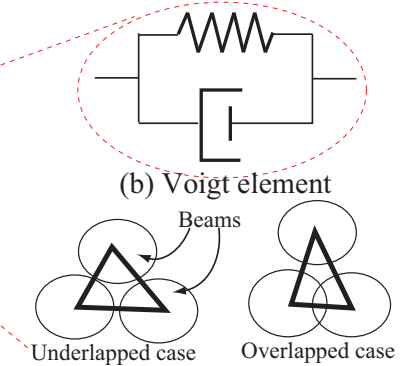

(c) Positions of beams
Fig. 5. Meshed contact area.

often than the latter, such that the coverage ratio of the entire contact area is always less than $100 \%$. The coverage would then vary, depending on how coarse or fine the contact area was meshed, with the maximum ratio reached with less superposition being $96 \%$. Thus, we can flexibly change the coverage ratio by varying the geometrical shape of the beams to adapt to specific simulations. Beams are constrained to the contact area on the skin, such that the movement of their free-ends would be helpful in assessing stick/slip events on the contact area during sliding motions. We have called this model the Beam Bundle Model (BBM).

\section{Avoiding Re-meshing the Contact Area at Different Con- tact Depths}

When a fingertip slides on a surface or several fingertips grasp an object, the contact depth may vary to adapt to the outside environment. In this scenario, the contact area meshed by finite elements must also be adjusted to have geometric characteristics that differ from those of the initial contact, significantly altering the number of virtual beams and their geometry. This would be inconvenient for programming and control, since it produces discontinuity during change of contact depth. We propose a method that permits one meshing, with no remeshing during changes in contact depth.

Since each node on the meshed contact area corresponds to one beam located exactly on this node, two groups of beams are present: contact beams positioned inside the contact, and non-contact beams that are outside the contact area. When contact depth varies, the following two conditions must be satisfied:

1) Contact beams at different contact depths must be unchanged, leaving their corresponding nodes unchanged.

2) Non-contact beams that become contact beams at the updated contact depth must be assigned a predetermined node on the contact pad.

We, therefore, only meshed the contact area at the possibly maximum contact depth, says $d_{\max }$. At this contact depth, the contact area can be meshed with a set of $N$ nodes $\left\{n_{i}, i=1: N\right\}$, and corresponding $N$ beams $\left\{b_{i}\right\}$. These nodes and beams are unchanged throughout different contact depths. At a specific contact depth $d<d_{\max }$, the position of each beam must be checked to determine whether the beam lies inside or outside the new contact area. Depending on its position, its corresponding node will be marked as a contact node $n_{j}^{c}\left(j=1: N_{c}\right.$, where $N_{c}$ is the total number of contact nodes), or a non-contact node $n_{k}^{n c}\left(j=1: N_{n c}\right.$, where $N_{n c}$ is the total number of non-contact nodes, and $N_{c}+N_{n c}=N$ ). Thus, there are two groups of nodes on the meshed area, $\left\{n_{j}^{c}\right\}$ and $\left\{n_{j}^{n c}\right\}$. We supposed that non-contact nodes were virtual nodes, which only exist to assure that the previously meshed area's geometrical characteristics are unchanged but have no effect on the deformation of the entire area or on the movements of contact nodes. To satisfy this condition, if at least one of the three vertices of a triangle on the meshed area is virtual, the triangle's stress-strain relation will be eliminated. In detail, the stress-strain relationship in a triangle can be converted into a relationship between a set of forces $\mathbf{f}$ applied to nodes and their displacements $\mathbf{u}$. This set of forces of a Voigt element is calculated as:

$$
\mathbf{f}=\mathbf{J}_{\lambda}\left(\lambda^{\text {ela }} \mathbf{u}+\lambda^{v i s} \dot{\mathbf{u}}\right)+\mathbf{J}_{\mu}\left(\mu^{e l a} \mathbf{u}+\mu^{v i s} \dot{\mathbf{u}}\right),
$$

where $\mathbf{u}$ and $\dot{\mathbf{u}}$ are the displacement and velocity vectors of nodal points, respectively, $\mathbf{J}_{\lambda}$ and $\mathbf{J}_{\mu}$ are connection matrices that depend solely on the geometric coordinates of nodes, and $\lambda^{\text {ela,vis }}$ and $\mu^{\text {ela,vis }}$ are Lame's constants that can be described by Young's modulus $E$, Poisson's ratio $\gamma$, and viscous modulus $c$. Thus, to eliminate the interaction of $\left\{n_{j}^{c}\right\}$ to $\left\{n_{j}^{n c}\right\}$, we simply set Lame's constants of a triangle that owns non-contact nodes to zero temporarily for this contact depth. If any $\left\{n_{j}^{n c}\right\}$ becomes $\left\{n_{j}^{c}\right\}$, its corresponding triangle's Lame's constants will recover their original values. As a result, simulations always run on one meshed contact area during change of contact depth, taking into account only real contact nodes and giving virtual nodes no stress/strain relations.

\section{E. Derivation of Motion Equation}

1) During Loading/Unloading: In this section, we derive a general equation for the normal deformation of one inhomogeneous beam constituted of a set of Voigt models connected in series, referred to as a generalized Voigt model.

Supposing that one beam consists of $n$ Voigt elements, then $\varepsilon$ would be the extensional strain of the beam while $\varepsilon_{i}$ would be the extensional strains of the $i$-th element. Extensions $\varepsilon$ and $\varepsilon_{i}$ through $\varepsilon_{n-1}$ are independent state variables, and $\varepsilon_{n}$ can be described dependently by other variables:

$$
\varepsilon_{n}=\varepsilon-\varepsilon_{1}-\ldots-\varepsilon_{n-1} .
$$

Let $E_{i}$ and $c_{i}$ be the elastic and viscous moduli of the $i$-th Voigt element. Noting that stresses generated by individual elements are equal to each other, we have:

$$
\sigma=E_{1} \varepsilon_{1}+c_{1} \dot{\varepsilon}_{1}=E_{2} \varepsilon_{2}+c_{2} \dot{\varepsilon}_{2} \ldots=E_{n} \varepsilon_{n}+c_{n} \dot{\varepsilon},
$$

where $\sigma$ indicates the generated stress by the deformation of the beam. Dividing the above equations by $c_{1}$ through $c_{n}$ 
and summing up all equations yields:

$$
\sigma=\frac{\left(\frac{E_{1}}{c_{1}} \varepsilon_{1}+\ldots+\frac{E_{n-1}}{c_{n-1}} \varepsilon_{n-1}\right)+\frac{E_{n}}{c_{n}}\left(\varepsilon-\varepsilon_{1}-\ldots \varepsilon_{n-1}\right)+\dot{\varepsilon}}{\frac{1}{c_{1}}+\frac{1}{c_{2}}+\ldots \frac{1}{c_{n}}}
$$

Note that using the above equation, stress $\sigma$ can be computed from state variables $\varepsilon_{1}$ through $\varepsilon_{n-1}$ and $\dot{\varepsilon}$. State variables satisfy the following differential equations:

$$
\dot{\varepsilon}_{1}=-\frac{E_{1}}{c_{1}} \varepsilon_{1}+\frac{1}{c_{1}} \sigma, \ldots, \dot{\varepsilon}_{n-1}=-\frac{E_{n-1}}{c_{n-1}} \varepsilon_{n-1}+\frac{1}{c_{n-1}} \sigma
$$

Consequently, we can construct a dynamic equation to calculate deformations of elements in an inhomogeneous beam. This process was repeated for all virtual beams in a fingertip. This derivation can also be utilized to model any inhomogeneous soft objects other than fingertips.

2) Sliding Motion: Utilizing the equations proposed in [9], we could derive equations for the sliding motion of a fingertip's meshed contact area. If a fingertip was pushed vertically onto a flat surface, then slid horizontally at constant speed, then virtual beams would deform correspondingly, including normal and bending deformations. Several assumptions may simplify this calculation:

1) Interactions between neighboring beams only occur at their free ends on the contact pad.

2) Only beams with free ends acting on the contact pad are considered. Beams outside the contact surface are irrelevant to the sliding motion of a fingertip.

3) The nail is completely rigid and experiences no deformation during the sliding motion of a fingertip.

4) The area of the nail is always larger than the contact area.

The first and the second assumptions are important for drastically reducing costs of calculation, since the tissue volume of a fingertip deforms uniformly, while the skin on the contact area stretches differently at different locations. Since the superposition of beams is small and unrelated to the precision of the model, beams deformed by pushing action do not affect beams positioned outside the boundaries of the contact area, making the second assumption sufficient for modeling deformations of an entire fingertip. Young's moduli of bone and nail are much larger than those of tissue and skin I. Thus the third assumption above is also acceptable for calculations without degrading the precision of the model. The fourth assumption represents reality, since most human fingertips possess a nail that covers almost the entire upper part of the fingertip. This eliminates beams on the contact area that make no contact with the bone and nail, including only those contacting the skin.

Using these assumptions, we were able to implement force calculation and motion equations. For a normal force, each beam that makes contact with a flat surface possesses a deformation $d_{n}^{i}$. As the external tangential force $F_{t}$ starts to activate, the fingertip has not yet slid. The contact surface still sticks to the plane, causing the fingertip to deform. At this time, the free ends of all contacting beams are bent with
TABLE I

PhysicAl PARAMETER FOR THE HuMAN Fingertip ([5])

\begin{tabular}{|c||cccc|}
\hline & Tissue & Skin & Bone & Nail \\
\hline \hline $\mathrm{E}[\mathrm{Pa}]$ & $3.4 \times 10^{4}$ & $1.36 \times 10^{5}$ & $1.5 \times 10^{9}$ & $4.3 \times 10^{9}$ \\
$\mathrm{c}[\mathrm{Pa} . \mathrm{s}]$ & 0.1 & 10 & 1 & Not used \\
$\gamma$ & 0.48 & 0.48 & 0.48 & Not used \\
\hline
\end{tabular}

the same bending strain $\delta s$, which can be calculated as [13]:

$$
\delta s=\frac{3 \mu F_{n}}{16 R} \frac{2-v}{G}\left\{1-(1-\Phi)^{2 / 3}\right\},
$$

where $\Phi=F_{t} / \mu F_{n}$ is the tangential force coefficient, $R$ is the radius of the fingertip, $\mu$ is the coefficient of friction, $v$ is Poisson's ratio, and $G$ is the modulus of shear elasticity. Due to assumption 1, above, bending of one inhomogeneous beam (such as the beams in group N-B-S) is regarded as the bending of the B-S beam. Thus its bending stiffness is calculated based only on the geometric quantities of the B-S beam. Equations for estimating normal and bending forces have been reported [14].

In an FE-based meshed contact area, there is a viscoelastic force at each contact node, which can be calculated based on that node's geometric characteristics, as shown in Eq. (1). We also introduced the law of friction onto the contact surface of each node. Its value changes based on its contact state, stick or slip, and calculated correspondingly:

$$
\mathbf{f}_{f r}^{i}= \begin{cases}\mathbf{f}_{v e}^{i}+\mathbf{f}_{b}^{i} & \text { if } \mathbf{f}_{v e}^{i}+\mathbf{f}_{b}^{i}<\mu \mathbf{f}_{n}^{i} \Rightarrow \text { Stick } \Rightarrow \mathbf{A}^{i}=\mathbf{A}_{1}^{i} \\ \mu \mathbf{f}_{n}^{i} & \text { if } \mathbf{f}_{v e}^{i}+\mathbf{f}_{b}^{i} \geq \mu \mathbf{f}_{n}^{i} \Rightarrow \text { Slip } \Rightarrow \mathbf{A}^{i}=\mathbf{A}_{0}^{i}\end{cases}
$$

where $\mathbf{A}_{2,2}^{i}$ be a matrix to describe the constraint of the $i$-th node.

Finally, by utilizing Lagrangian formula and Constraint Stabilization Method (CSM) with a set of Lagrangian constraints $\lambda$ and a pre-determined angular frequency $\omega$, we were able to construct motion equations of all contacting nodes on the contact pad as follows [9]:

$$
\left(\begin{array}{ccc}
\mathbf{I} & \mathbf{0} & \mathbf{0} \\
\mathbf{0} & \mathbf{M} & -\mathbf{A} \\
\mathbf{0} & -\mathbf{A}^{T} & \mathbf{0}
\end{array}\right)\left(\begin{array}{c}
\dot{\mathbf{u}}_{N} \\
\dot{\mathbf{v}}_{N} \\
\lambda
\end{array}\right)=\left(\begin{array}{c}
\mathbf{v}_{N} \\
-\mathbf{K}_{e l a} \mathbf{u}_{N}-\mathbf{K}_{v i s} \mathbf{v}_{N}+\mathbf{F} \\
\mathbf{A}^{T}\left(2 \omega \mathbf{v}_{N}+\omega^{2} \mathbf{u}_{N}\right)
\end{array}\right)
$$

where $\mathbf{M}$ is the inertia matrix of the 2-D FEA contact pad, $\mathbf{F}$ is the vector of external forces such as bending force and friction force. This equation is linear and solvable since the matrix is regular, implying that we can compute $\dot{\mathbf{u}}_{N}$, and $\dot{\mathbf{v}}_{N}$, which are displacements and velocities of contacting nodes. Details of this derivation can be seen in [9].

\section{Simulation AND VerificAtion}

In this simulation, the fingertip was pushed vertically at a pre-determined contact depth, and with a tangential movement at constant velocity. Parameters used for simulation are summarized in Tables I. To validate this model, we performed an experiment similar to that in Section II for an artificial fingertip. We created an artificial inhomogeneous 


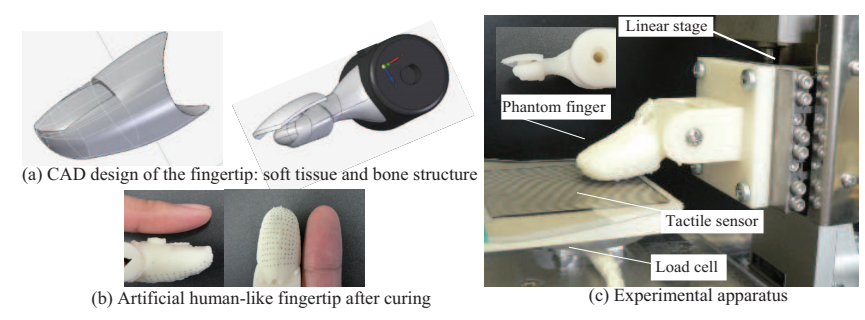

Fig. 6. CAD model of the human-like fingertip and the experimental apparatus.

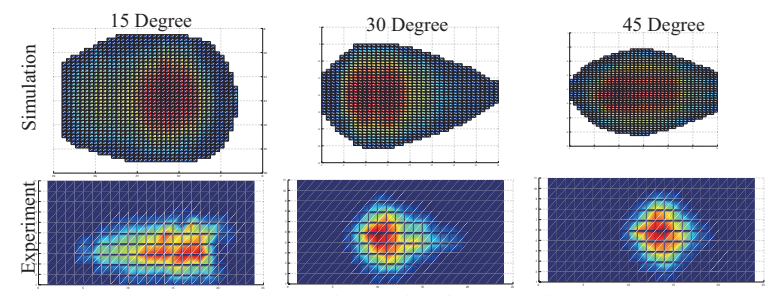

(a) Normal force distribution on the contact area

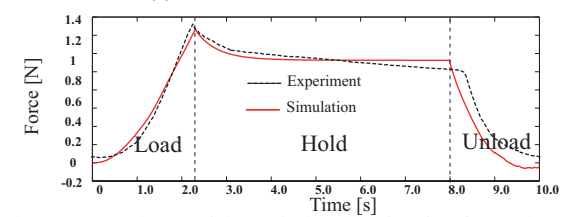

(b) Dynamic response of normal force during simulated and experimental load/unload test

Fig. 7. Comparison of normal force distribution from simulation (upper row) and experiment at different contact angle. The graph show a dynamic response of the total normal force during simulated load/unload test.

human-like fingertip based on MRI data, with surfaces interpolated and smoothed by a computer-aided design program (see Fig. 6). The bone structure and nail were made with a 3-D printer, while the soft tissue, with softness similar to that of human tissue, obtained after curing a polyurethane rubber gel in a designed mold. Utilizing this finger, we were able to precisely validate the proposed model. To create movements for the fingertip, we attached it onto a 2-D linear motorized stage that can provide $2 \mu \mathrm{m}$-in-resolution step.

\section{A. Force-Related Results}

Fig. 7(a) shows the normal force distribution when the fingertip was pushed at a contact depth of $2 \mathrm{~mm}$ and at different angles of contact. We found that the maximum force area was around the tip close to the nail, while smaller values of normal forces were distributed mostly at the boundary of the contact surface. When inclination contact increases, the maxima shift from right to left. Similar results were observed experimentally. Thus, although the outer shape of a human fingertip is likely symmetrical and even, the force distribution is not identical to that of hemispheric homogeneous fingertip. The inner distribution of distal phalanxes causes remarkably different geometric shapes, as well as non-uniform deformations of beams over the contact area. Fig. 7(b) illustrates one simulated dynamic load/unload test of normal force response. Exponential growth and decay of the normal force occurred during the loading and unloading phases, respectively. Also
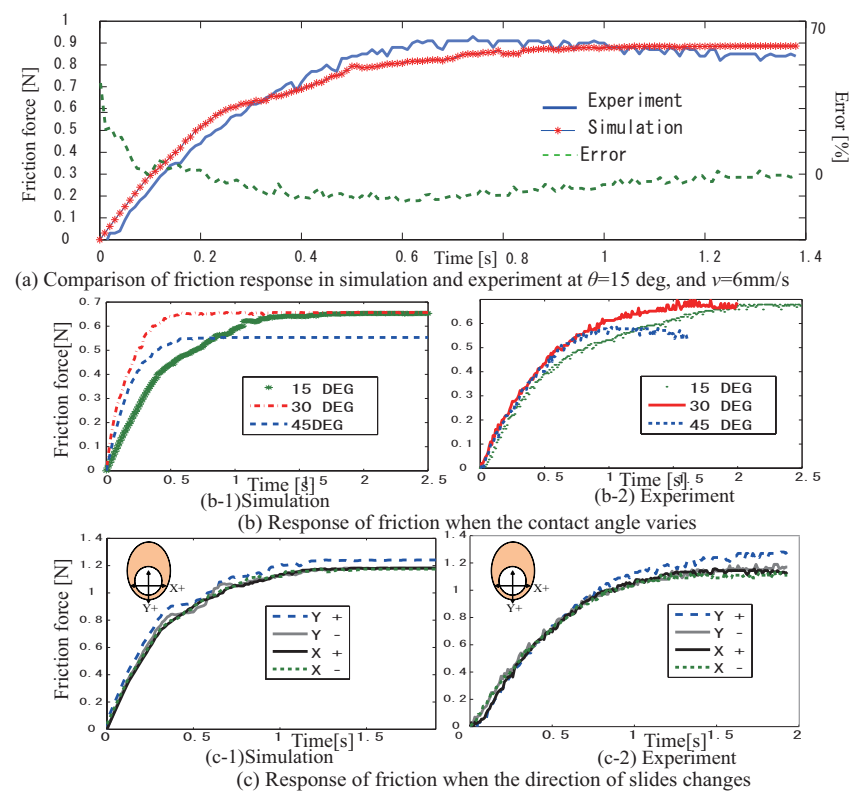

Fig. 8. Friction responses in various conditions of slide.

the relaxation of the normal force when the fingertip was held at a given contact depth showed complete agreement with those described in Section II-A.

Fig. 8(a) plots shows the response of friction force during the transition from stick to slip phase at constant speed. Similar to findings in Section II-B, two phases featuring changes in friction forces were easily observed. Stick phase represents a pre-sliding phase, when a fingertip begins to move but the contact area still sticks to the surface, i.e. the fingertip has not moved sufficiently to markedly increase the force of friction, making it almost linear. Slide phase indicates the total movement of the contact area, with the friction force remaining unchanged at a given speed. Fig. 8(a) also shows the agreement between simulation and experimental results, with only the error being significantly different at the initial time, declining to less than $10 \%$ during the transition. The deflection observed at simulation results were caused by an artifact of numerical simulation. Plots in Fig. 8(a) indicate that, when the contact angle of inclination varies at the same contact depth and sliding velocity, the duration of the stick phase will also change correspondingly, with a smaller angle associated with a larger duration of the stick phase. Similar findings were observed experimentally. At a small contact angle the contact area becomes wider, resulting in more time for localized displacement to occur. Moreover, friction would be greater at the same contact depth and sliding speed. We also investigate changes in friction response when a fingertip was moved in four different directions. We found that can observe in Fig. 8(c), during both simulations and experiments, friction did not vary significantly over the four directions Fig. 8(c). Friction along the $Y+$ direction is always slightly greater than friction along the remaining directions, making it difficult to assess directions of slide based solely on frictional responses. 

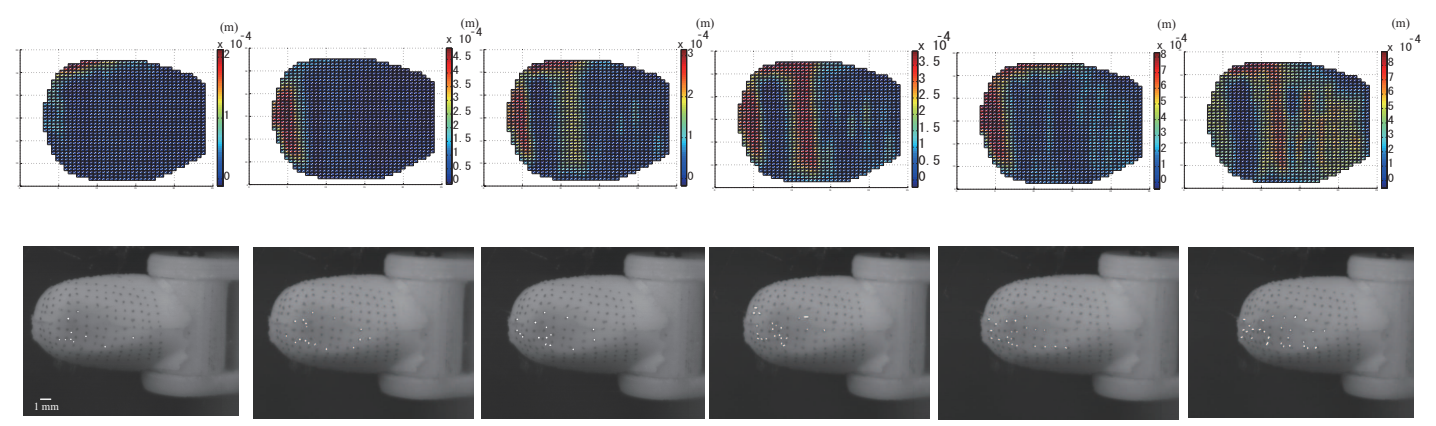

Fig. 9. Comparison in term of local displacement distributions over time when the fingertip slides along $Y$ directions. Upper row is results from simulation.
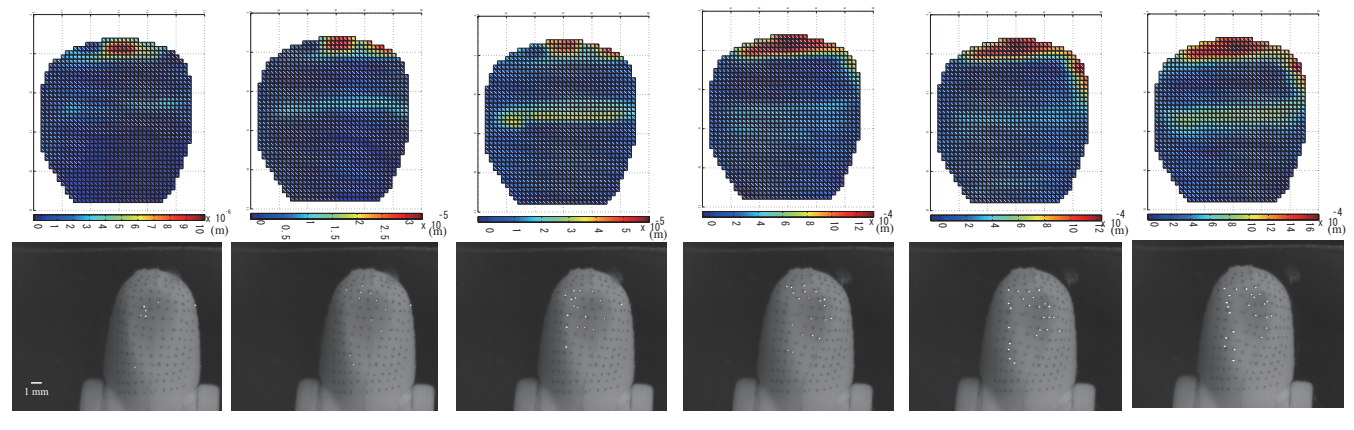

Fig. 10. Comparison in term of local displacement distributions over time when the fingertip slides along $X$ directions. Upper row is results from simulation.

\section{B. Localized Displacement Phenomenon (LDP) during Pre- Slide Phase}

One noticeable result was the success of representations of localized displacements on the contact area during pre-slide phase during simulation. Fig. 9 illustrates the distribution and localized movements of nodes contacting the contact area over time during the pre-slide phase. Bright and hot color zones indicated greater movement than cold and dark color zones. These distribution were calculated from the simulation of a sliding trial with a sliding speed of $2 \mathrm{~mm} / \mathrm{s}$, a coefficient of friction of 0.7 , and a contact depth of $2 \mathrm{~mm}$. We found that displacement occurred initially near the boundary of the contact area, followed by the gradual displacement along the direction of flow. Moreover, other propagations are those of movements spreading from outer to inner areas. Thus, propagation of displacement relies on a normal force distribution, with smaller forces distributed mainly near the boundary than in the middle part of Teaneck (see Fig. 7(a)). Gross sliding occurs only when partial slippage is present throughout the contact pad, a result totally coincidental to the results of the investigation, as shown in Section II-C.

Fig. 9 and 10 illustrate partial movements of contacting areas on a contact pad during the pre-slide phase, with movements obtained from both simulations and experiments along two directions. While the movements of contacting points in an experiment are indicated by white bars obtained from optical tracking [12]; a hot color during simulation may represent larger displacement areas on the contact pad (note that the color scale is different over images). We found that the propagation of slippage on the contact pad during both simulations and experimentally shows obvious similarities. Moreover, we found that the propagation of localized slippage varies significantly when the fingertip slides along different directions, similar to the conclusion mentioned in Section IV-B. As a result, even responses of friction over the direction of the slides did not vary significantly (Fig. 8(c), indicating that the localized displacements act differently. This phenomenon, while complicated for robotics applications, is easily sensed by the high density of mechanoreceptors underneath the skin in our model of human fingertips. This ability may explain why humans act so comfortably during the incipient slipping of a grasped object when it tends to slip out of hand. Mechanoreceptors are unable to change the frictional force that predicts incipient slippage. These receptors are stimulated by local displacement or stretching of the skin. Tactile signals are sent to the brain for processing; and commands are transmitted to motor systems to apply more force to prevent the slippage in a real-time scenario [15]. Modeling and assessing localized displacement phenomena makes it easier to understand the underlying mechanism of tactile perception of human fingertips during sliding motion.

Consequently, we have verified the BBM in an artificial human-like fingertip using a fine experimental setup. Most of mechanical characteristics of artificial fingertips were having by matching those of simulated model, such as the friction and the localized displacement phenomenon.

\section{Discussion}

\section{A. Significance of the LDP in Tactile Sensing}

We found that our proposed BBM may be able to model inhomogeneous soft fingertips, thus determining how and 
when slippage occurs during the stick phase through propagation of localized displacements on the contact area. These findings may be helpful in intuitively imaging the phenomena that dominate the characteristics of sliding contacts. Humans never feel the exact friction force that acts on the contact area to judge tactile feelings. Instead, mechanoreceptors underneath the skin are stimulated by skin deformation/stretch through stress or vibration, then transferred to the brainstem along axonal processes (see [15]), allowing tactile sensations to be interpreted. This supports our idea of utilizing the LDP to detect incipient slip in a tactile sensing system. It states that if a sensing system can detect the propagation of slippage on the contact area during the stick phase, in a direct or indirect way, the incipient slippage can be judged in a proper and timely manner. One direct method is to use high speed cameras to track movement at each contact point, intuitively showing where the contact patch is or is not sliding. Nonetheless, this method is not suitable for embedding in any system due to its size. Therefore, a novel sensing system is needed for ease of fabrication, a system conforming to the LDP indirectly. Any indirect method must consider localized displacements into transduced outputs, allowing robots/machines to assess when slippage is about to occur.

\section{B. Beam Bundle Model}

We have described here a process for using the BBM to model various types of human-like fingertips, from actual to artificially designed nodules, and from homogeneous to inhomogeneous types. The main characteristics of a sliding human fingertip were successfully reflected in the simulated model, especially the localized displacements during preslide phase. The proposed method can act as a platform for studying the sliding mechanics of sliding robotic fingertips and of inhomogeneous soft objects as a whole, in tactile perception, or in creating models in a virtual environment in haptic research. Various friction models have utilized contact nodes, such as the LuGre and Dahl [16] models, rather than the Coulomb friction model. In addition, our model was convenient for calculating the moment of friction generated through sliding motion by synthesizing partial friction moments of contact nodes to a pre-determined axis. We are planning to create a bigger database of human fingertips, ranging from children to adults at different ages, to further investigate differences in sliding tactile mechanism, as well as to enhance the proposed BBM. This model can be employed to model grasping/manipulation with coefficient frictions similar to those of humans and humanoid robots.

All codes were implemented in a Microsoft Visual Studio $\mathrm{C}++$ environment, in a common Personal Computer with Intel Core Duo 3.0 GHz chipset and 2.0 GB RAM. A typical dynamic simulation trial of $2 \mathrm{sec}$ took about 24 minutes to complete. Although this was far from a real-time application, faster performance may be achieved by implementing it on a GPU-equipped computer, and we are currently working toward it.

\section{CONCLUSION}

We have investigated the theoretical sliding motion of a human fingertip model, focusing on the pre-slide phase, and utilizing our proposed BBM and MR images. Simulation results help us to assess the change of force, and especially localized displacement phenomena on the contact area during the pre-slide phase. Knowledge about this phenomenon will assist us in understanding the role of the skin in the recognition of slippage, as well as to develop a sensing system that detects incipient slippage of human fingertips, as well as being crucial for stable manipulation.

\section{REFERENCES}

[1] A. Nahvi, J. M. Hollerback, R. Freier, and D. D. Nelson, Display of Friction in Virtual Environments Based on Human Finger Pad Charactersistics, Proceedings of ASME Dynamic Systems and Control Division, pp. 179-184, 1998.

[2] N. Xydas, M. Bhagavat, and I. Kao, Study of soft-finger contact mechanics using finite analysis and experiments, IEEE Int. Conf on Robotics and Automation, vol. 3, pp. 2179-2184, April 2000.

[3] K. Dandekar, B. I. Raju, M. A. Srinivasan, 3-D Finite-Element Models of Human and Monkey Fingertips to Investigate the Mechanics of Tactile Sense, Transaction of the ASME- Journal of Biomechanical Engineering, Vol. 125, pp. 682-691, Oct. 2003.

[4] F. Barbagli, A. Frisoli, K. Salisbury, and M. Bergamasco, Simulating human fingers: a Soft Finger Proxy Model and Algorithm, 12th International Symposium on Haptic Interfaces for Virtual Environment and Teleoperator Systems, pp. 9-17, 2004.

[5] Z.K. Wang, L. Wang, V. A. Ho, S. Morikawa, and S. Hirai, A $3 D$ Non-Homogeneous FE Model of Human Fingertip Based on MRI Measurements, IEEE Transaction on Instrumentation and Measurement, Vol. 61, No. 12, pp. 3147-3157, Dec. 2012.

[6] S. Shimawaki, and N. Sakai, Quasi-static deformation analy- sis of a human finger using a three-dimensional finite element model constructed from CT images, Journal of Environment and Engineering, vol. 2, pp. 56-63, 2007.

[7] I. Kao and M. R. Cutkosky, Quasistatic Manipulation with Compliance and Sliding, The Int. Jour. of Robotic Research, Vol. 11, No. 1, pp. 20-40, Feb., 1992.

[8] C. Duriez, F. Dubois, A. Kheddar, and C. Andriot, Realistic Haptic Rendering of Interacting Deformable Objects in Virtual Environments, IEEE Trans. on Visualization and Computer Graphics, Vol. 12, No. 1, pp. 36-47, 2006.

[9] V. A. Ho and S. Hirai, Understanding Slip Perception of Soft Fingertips by Modeling and Simulating Stick-Slip Phenomenon, Robotics: Science and Systems VII, Los Angeles, CA, US, June 2011.

[10] E. R. Serina, C. D. Mote Jr., and D. Rempel, Force Reponse of the Fingertip pulp to Repeated Compression-Effects of Loading Rate, Loading Angle and Anthropometry, J. Biomechanics, Vol. 30, No. 10, pp. 1035-1040, 1997.

[11] D. L. Jindrich, Y. Zhou, T. Becker, J. T. Dennerlein, Non-linear Viscoelastic Models Predict Fingertip Pulp Force- Displacement Characteristics during Voluntary Tapping, J. Biomechanics, Vol. 36, pp. 497$503,2003$.

[12] M. Tada and T. Kanade, An Imaging System of Incipient Slip for Modeling How Human Perceives Slip of a Fingertip, The 26th Annual Intl. Conf. of the IEEE EMBS, pp. 2045-2048, San Francisco, Sept., 2004.

[13] R. D. Mindlin, Compliance of elastic bodies in contact, Trans. ASME, J. Appl. Mech., vol. 16, pp. 259-268, 1949.

[14] S. Timoshenko and J. N. Goodier,Theory of Elasticity, Mc-Graw-Hill, New York, 1970.

[15] M. J. Hertenstein, S. J. Weiss The Handbook of Touch, Springer Publishing Company, New York, 2011.

[16] K. J. Astrom and C. Canudas-de-wit, Revisiting the Lugre Friction Model, IEEE Control Systems Magazine, Dec. 2008, pp. 101-114. 